
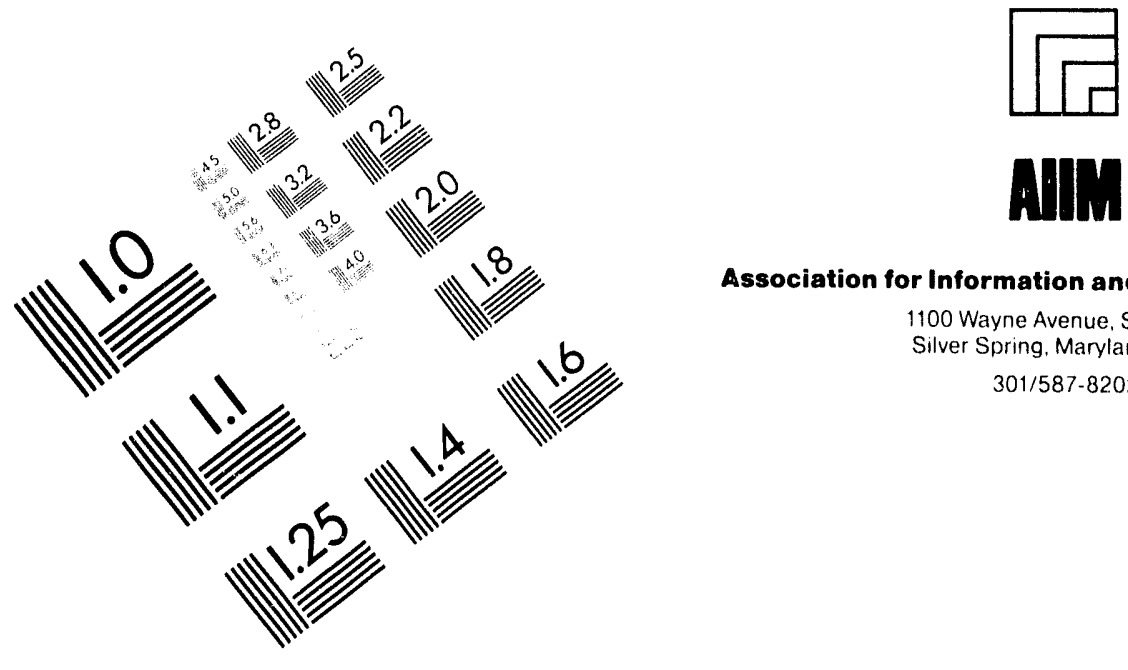

Association for Information and Image Management

1100 Wayne Avenue, Suite 1100

Silver Spring, Maryland 20910

301/587-8202

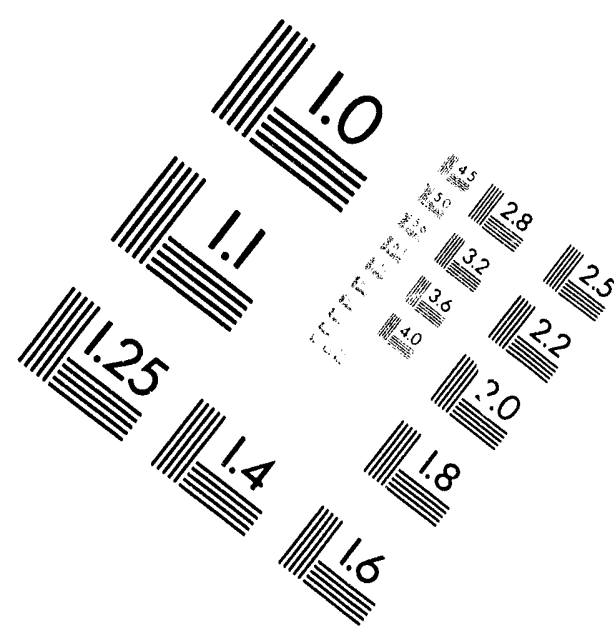

\title{
Centimeter
}

$\begin{array}{llllllllllllllll}1 & 2 & 3 & 4 & 5 & 6 & 7 & 8 & 9 & 10 & 11 & 12 & 13 & 14 & 15 & \mathrm{~mm}\end{array}$

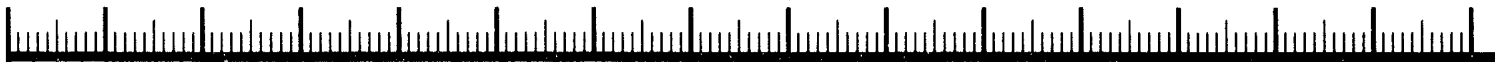
Inches
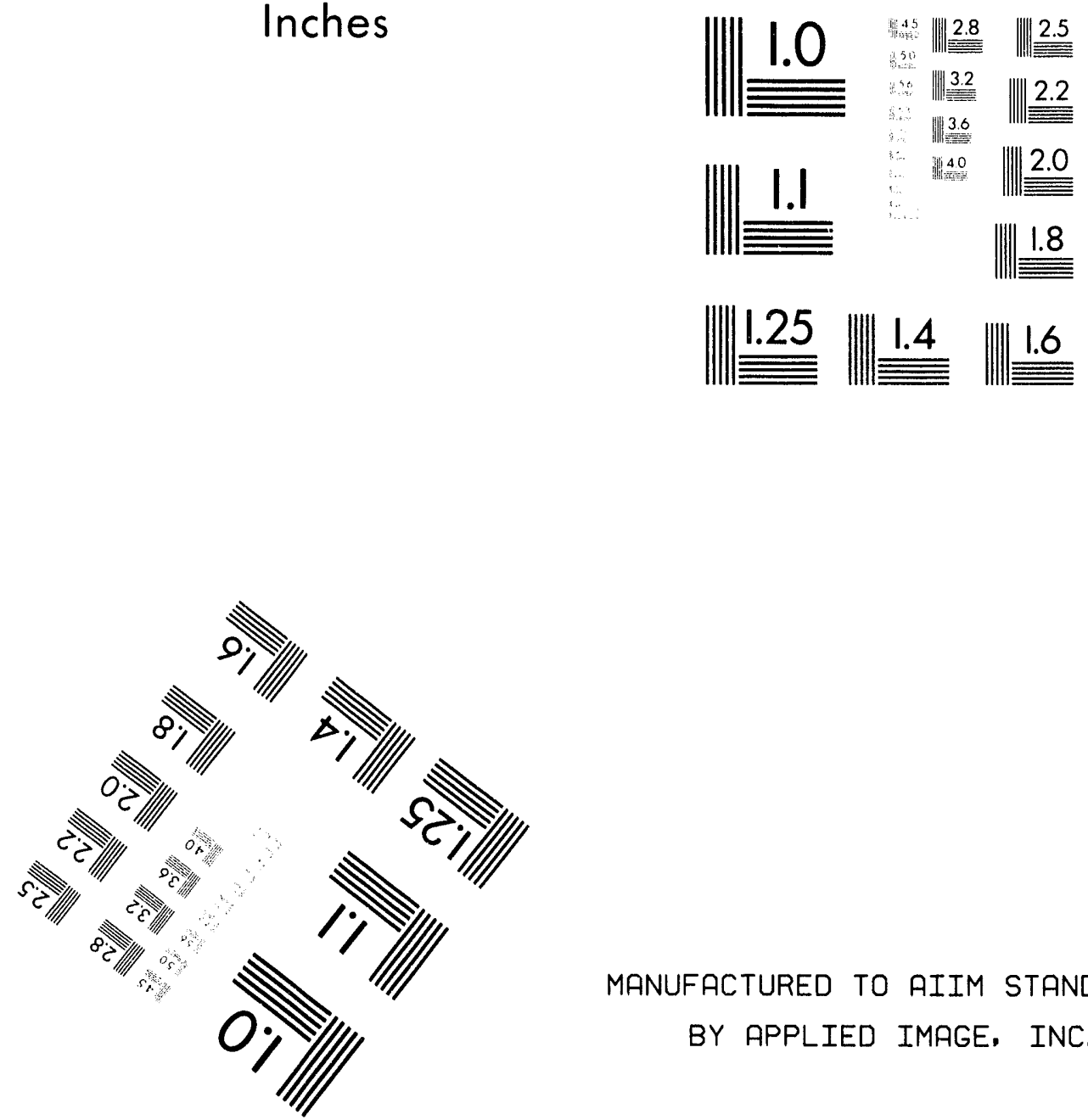

MANUFACTURED TO AIIM STANDARDS

BY APPLIED IMAGE, INC.

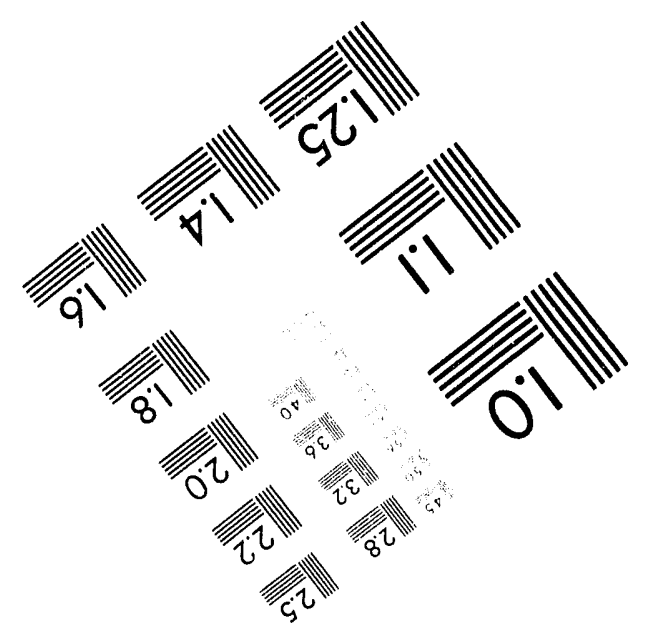



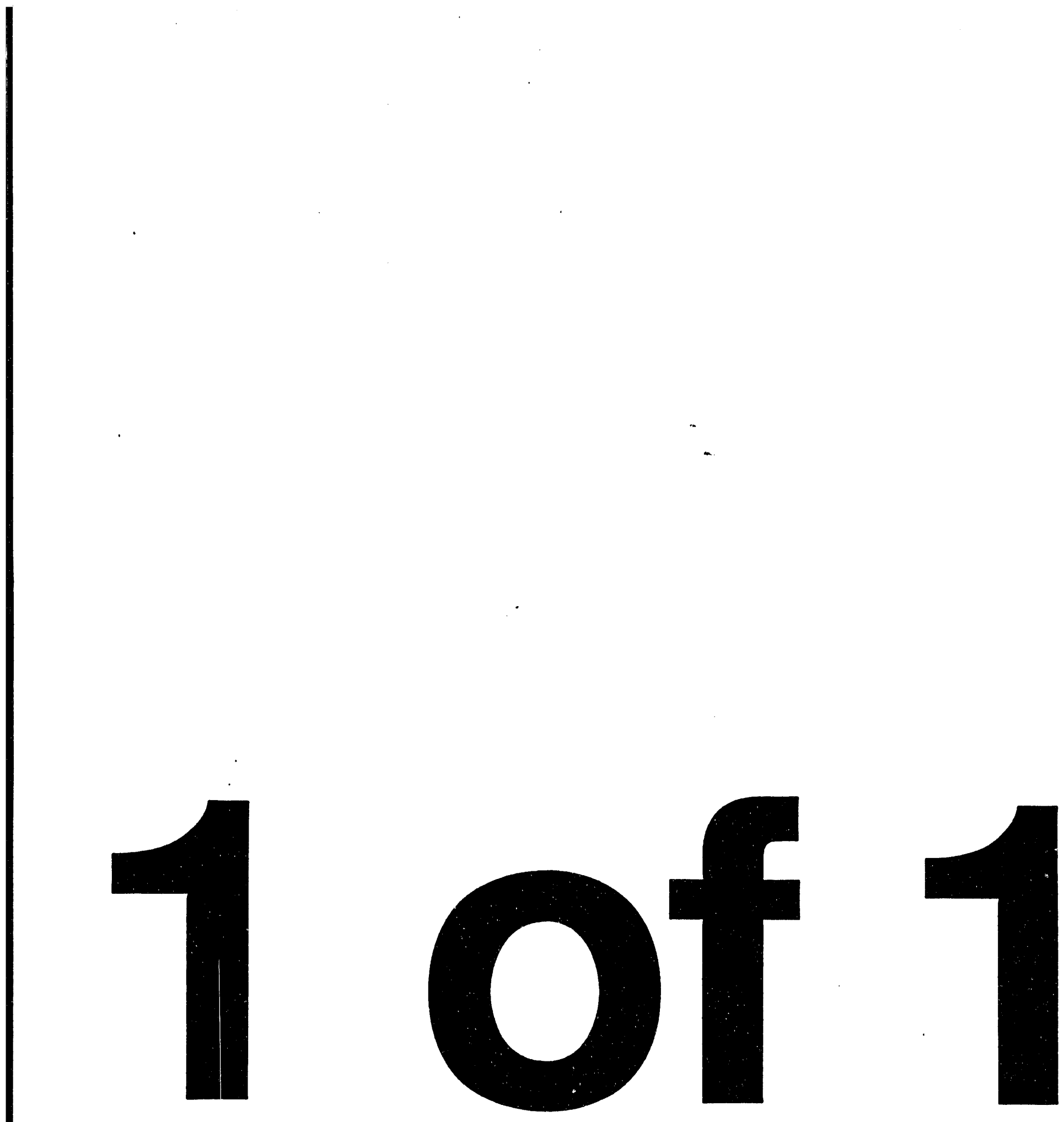
LBL -35236

\section{Statistical Multifragmentation: Is the Distinction Between Simultaneous and Sequential Decay Inessental?}

Presented at the Gross Properties of Nuclei and Nuclear Excitations International Workshop
XXII, Hirschegg. Kleinwalsertal; Austria January $17-22,1994$

L.G. Moretto, D.N. Delis, and G. J. Wozniak,

Lawrence Berkeley Laboratory, University of California, Berkeley, CA 94720, USA

January 1994

This work was supported in part by the Director, Office of Energy Research, Division of Nuclear Physics of the Office of High Energy and Nuclear Physics of the U.S. Department of Energy under Contract DE-AC0376SF00098. 


\title{
Statistical Multifragmentation: Is the Distinction Between Simultaneous and Sequential Decay Inessential?
}

\author{
L.G. Moretto, D. N. Delis, and G. J. Wozniak \\ Nuclear Science Division, Lawrence Berkeley Laboratory \\ 1 Cyclotron Road, Berkeley, CA 94720
}

\begin{abstract}
A powerful method is devised to verify the statistical competition between two-, three-, four-, and n-body decays. It is shown that under rather general conditions, the simultaneous n-body multifragmentation probability can be reduced to the $n-1$ steops sequential probability.
\end{abstract}

\section{Introduction}

Recently, some experimental work has succeeded in isolating and characterizing what appear to be true multifragmentation sources formed in reverse kinematics reactions ${ }^{1,2}$. These sources are formed in a process akin to incomplete fusion, whereby one partner of the collision picks up, and fuses with, a variable portion of the other partner. From the kinematics of the event, it is possible to determine how much mass has been picked up and what is the excitation energy associated with the fused object ${ }^{3}$. Surprisingiy, these sources, once characterized as described above, undergo multifragment decay in a way that is singularly independent of the formation process. The observed branching ratios for binary, ternary, quaternary, and quinary decays seem to depend almost exclusively upon the excitation energy $E$ of the fused object, and remarkably little upon the target-projectile combination or even the bombarding energy ${ }^{2}$.

The obvious question that we want to address is: what is the multi-fragmentation mechanism of these sources? In particular, is this decay controlled by dynamics, or by statistics?

\section{An Empirical Test for Statistical Branching Ratios}

To answer this question, one can examine the data themselves in order to see whether they. contain signatures that may be brought forth without the help, or impediment, of any given model.

Let us suppose that the hot nuclear system formed in the heavy-ion reaction decays statistically, and that a barrier of some sort governs this decay. Alternatively, in the framework of the chemical equilibrium picture, one can consider the potential energy of each configuration as a barrier. It is conceivable that, in these pictures, there might arise a hierarchy of "barriers" such that all the binary configurations would have barriers closer to each other than to those of the ternary configurations, and so on. Thus, let us assume that $B_{2}, B_{3}, \ldots B_{n}$ are the average "barriers" associated with binary, ternary, and $n$-body decays. 
The decay probability for each channel should be proportional to the level density of the system $\rho(E)$ (dominated by the internal degrees of freedom) at an excitation energy equal to the available energy minus the barrier:

$$
P_{n}(E) \propto \rho\left(E-B_{n}\right)
$$

For a Fermi gas level density, we have

$$
P_{n}(E) \propto e^{2 \sqrt{a\left(E-B_{-}\right)}}
$$

where $a$ is the level density parameter.

For $E \gg B_{n}$ one obtains:

$$
P_{n}(E) \propto e^{2 \sqrt{a E}} e^{-B_{n} \sqrt{a / E}} \propto e^{-B_{n} / T} .
$$

For convenience, we want the ratio of the $n$-fold events to the binary events:

$$
\ln \left[P_{n} / P_{2}\right] \propto-\sqrt{a / E}\left(B_{n}-B_{2}\right)
$$

Thus, a plot of $\ln \left(P_{n} / P_{2}\right)$ vs. $E^{-1 / 2}$ should give a straight a line.

\section{The Experimental Verdict}

This simple theoretical prediction has been empirically verified for the overall fission probabilities in the $\mathrm{Pb}$ region, and used to prove that the rapid rise of the fission cross section in $\mathrm{e}^{-}$induced fission of similar nuclei is due to statistics. To see whether a similar dependence exists in the multifragmentation branching ratios, we have performed an experiment with the specific purpose of determining the multifragment branching ratios as a function of the excitation energy of the decaying source. The decay of the hot nuclear systems formed in ${ }^{197} \mathrm{Au}$-induced reactions was studied, following closely the approach of Ref. 2 , by determining the ratio of the $n$-fold events $(n=3,4$, and 5) with respect to the 2-fold events as a function of the excitation energy $E$. In the incomplete-fusion model ${ }^{4}$, the excitation energy is approximately related to the parallel component $V_{s}^{\prime \prime}$ of the source velocity $V_{s}$ by $E=E_{b}\left(1-V_{s}^{\prime \prime}\right.$ $\left./ V_{b}\right)$ where $E_{b}$ is the bombarding energy and $V_{b}$ is the beam velocity. This formula does not take into account preequilibrium emission, thus the calculated value of the excitation energy should be regarded as an upper limit.

The number of binary and multibody events was determined for different bins of the source velocity and thus of the excitation energy of the source. By this procedure, we obtained the probabilities for ternary, quaternary, and quinary decays, as a function of the calculated excitation energy, shown figure 1a. The measured probabilities were then corrected for the detection efficiency (see figure $1 \mathrm{~b}$ ). 


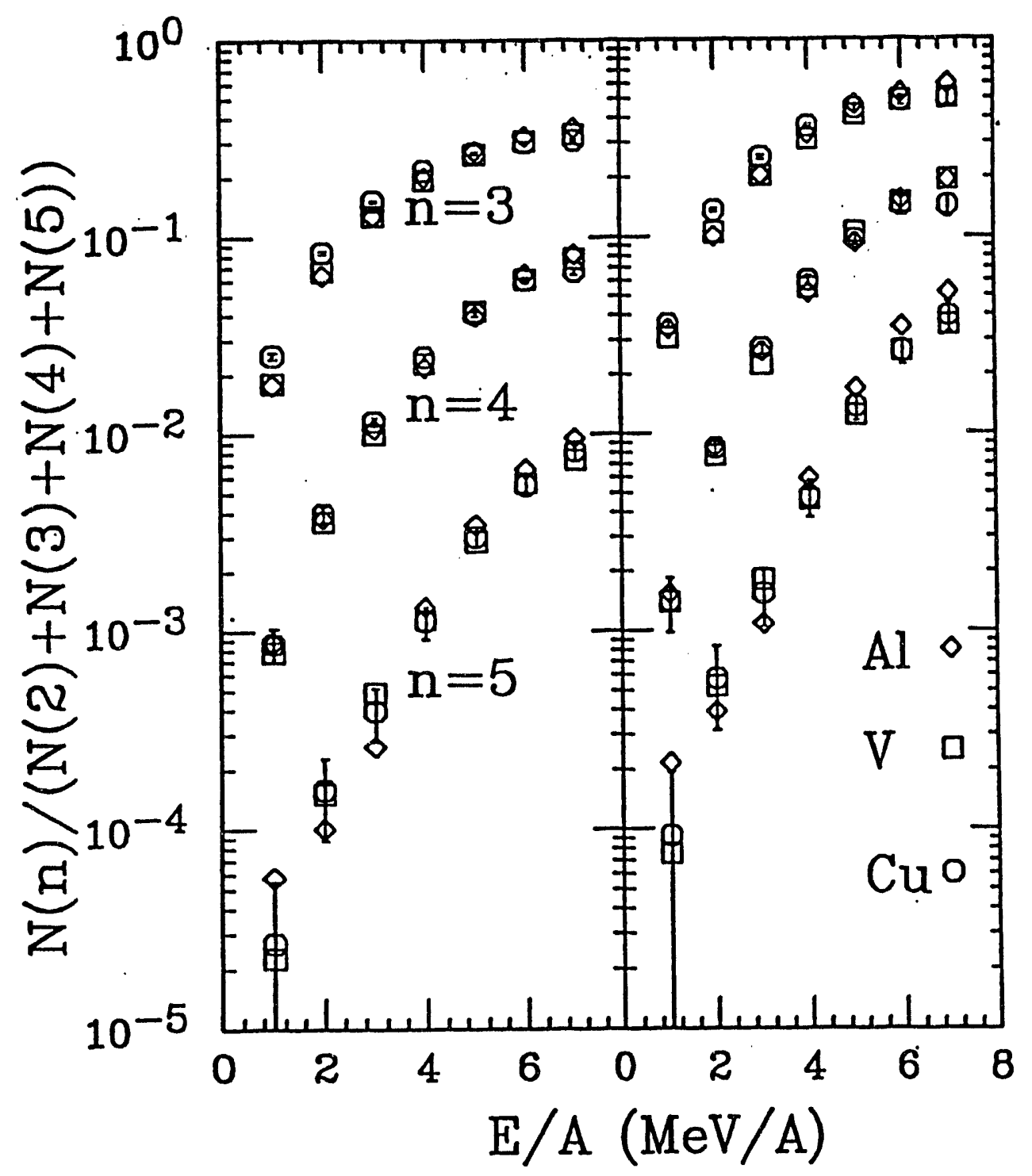

XBL $9310-1444$

Figure 1 a) Uncorrected relative probabilities for the ternary, quaternary, and quinary decays as a function of the source excitation energy for the $60 \mathrm{MeV} / \mathrm{u}$ ${ }^{197} \mathrm{Au}+{ }^{27} \mathrm{Al},{ }^{51} \mathrm{~V}$, and nat $\mathrm{Cu}$ reactions. b) Same as in a) after efficiency corrections (see text). Statistical errors are shown for the $\mathrm{Cu}$ target when they exceed the size of the symbols. 


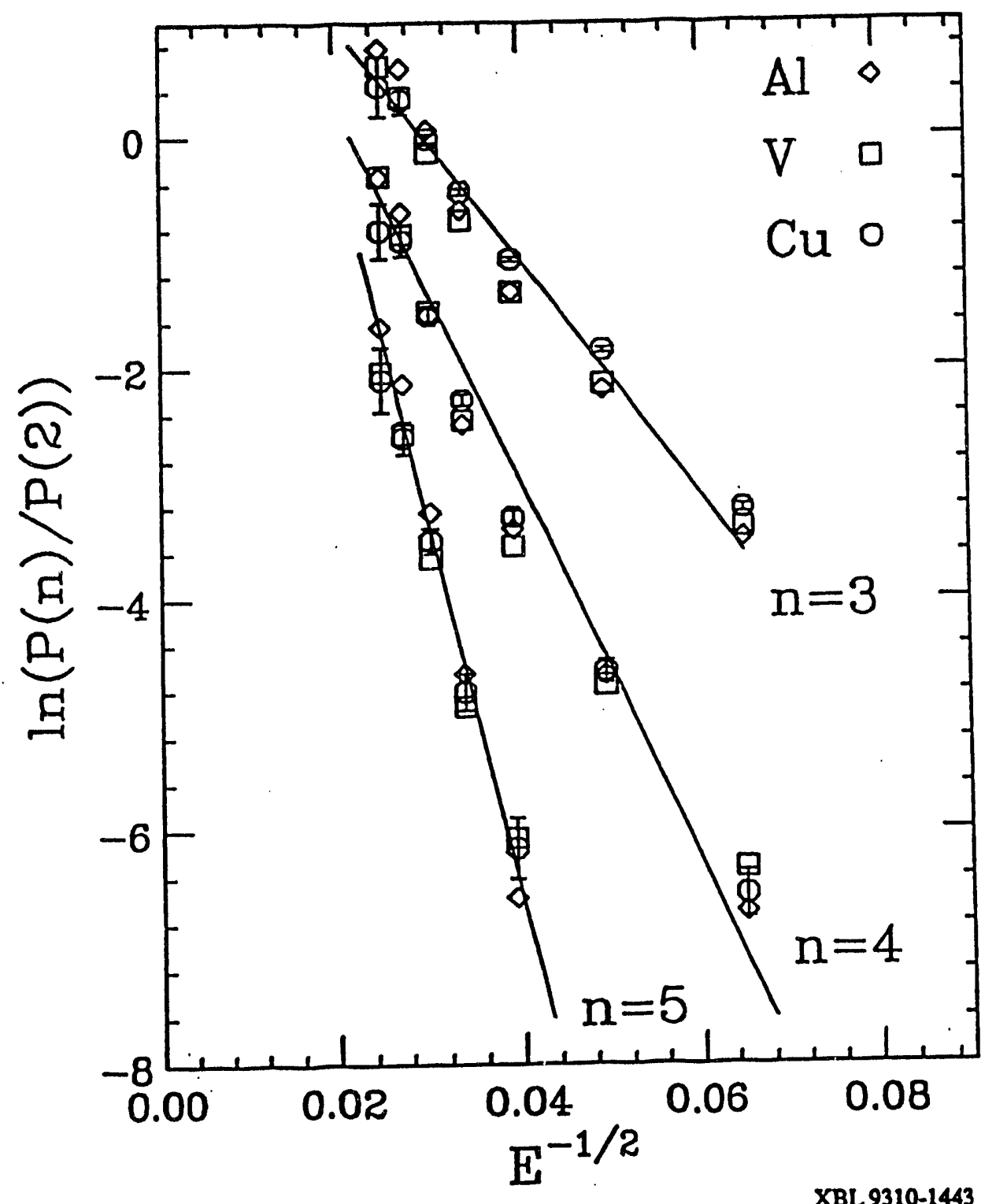

Figure 2. The natural logarithm of the ratio of the corrected 3-, 4-, and 5-fold probabilities to the 2-fold probability(symbols) as a function of $E^{-1 / 2}$ for the 60 $\mathrm{MeV} / \mathrm{u}{ }^{197} \mathrm{Au}+{ }^{27} \mathrm{Al},{ }^{51 \mathrm{~V}}$, and nat $\mathrm{Cu}$ reactions. The lines are the best fits to the data. Statistical errors are shown for the $\mathrm{Cu}$ target when they exceed the size of the symbols. 
The first striking observation is that the data from all the targets fall on the same curves. This is a strong confirmation of the results obtained for the La-induced reactions ${ }^{1,2}$. More specifically, once the multifragmentation source is characterized in terms of the kinematically determined excitation energy, the branching ratios for the various multifragment channels seein to be fixed and independent of the specific reaction that has produced the source. This shows that a decoupling between entrance and exit channel exists, leading, possibly to a "statistical" kind of decay.

This statistical feature is brought forth by the $E^{-1 / 2}$ plot shown in figure 2, which indeed generates straight lines. Similar straight lines are obtained from the La data ${ }^{5}$. We believe that the observed linear dependence for both the Au- and La- induced reactions is a strong signature for processes controlled by phase space. Since this dependence demonstrates statistical equilibrium between "different" channels, it may be deemed more significant evidence for deep equilibration than the thermalization of the kinetic energy spectrum within a given channel.

\section{Reducibility Theorem}

Does this approach tell us anything about whether the decay is simultaneous or sequential? It will be shown here that, under a fairly unrestrictive set of assumptions, the simultaneous n-body multifragmentation probability can be reduced to the $n-1$ sequential step probability.

Let us assume that:

1) The multifragmentation barrier $B_{n}$ can be written as follows:

$$
B_{n}=m B, \quad \text { where }(m=n-1) \text {. }
$$

2) All sequential barriers are the same and equal to $B$.

3) $p=\exp [-B / T]<<1 \quad(T=$ constant $)$

We will prove that the simultaneous multifragmentation probability $P_{n} \operatorname{sim}$ is equal to the sequential multifragmentation probability $P_{n}$ seq:

$$
P_{n}^{\text {sim }}=P_{n}^{\text {seq }}
$$

Assumption 2) can be easily verified, within the liquid drop model, for systems that are not too heavy, and for a broad range of mass asymmetries. Assumption 1) is approximately true for saddle shapes obtained by juxtaposing $\mathbf{n}$ equal spherical fragments, provided that the Coulomb force is negligible.

The proof is straightforward. For the simultaneous decay we have: 


$$
P_{n}^{\operatorname{sim}}=\exp \left[-B_{n} / T\right]=\exp [-m B / T]
$$

For the sequential case we have:

$$
P_{n}^{s e q}=(1 \cdot 2 \cdot 3 \cdots \cdot m) p^{m} / m !
$$

where the Poisson probability has been multiplied by a factor allowing for the various fragments to undergo binary decay.

This proves that there is no essential difference between simultaneous and sequential binary decay within the framework of the statistical model.

\section{The Mystery of the Mass Distribution (and of its moments)}

One of the most intensively studied aspects of multifragmentation is the fragment mass distribution and its moments. Several theories have been advocated in order to interpret such distributions. Among these are the liquid-vapor equilibrium at or near the critical point, a variety of versions of chemical equilibrium thcories, percolation models, sequential binary decay theories, etc.

The surprising result of this rather intensive exercise is that all the theories, with suitable choices of parameters, can fit the data with remarkable accuracy. This is all the more puzzling because of the different ingredients contained in the various theories. For instance some theories contain potential energies associated with the fragments and their interactions, while others do not. This pervasive success, contrary to the claims of the various authors, does not prove that any single theory is correct; in fact it allows one to eliminate from the game all the theories that contain any specific nuclear information, and to keep those that contain the least physical information. These theories are the percolation theories and others like that of Ploszajczak, which is based on sequential binary splitting with inhibition.

The meaning of this is still not apparent. However, it is not too risky to venture the guess that multifragmentation and the associated mass distribution is asymptotically combinatorial. If this is the case, the various theories that survive the minimum information test should be mappable one into the other. This may be the last task for mass distribution students.

\section{References}

1. Y. Blumenfeld et al., Phys. Rev. Lett. 66 (1991) 576.

2. P. Roussel-Chomaz et al., Nucl. Phys. A551 (1993) 508.

3. N. Colonna et al., Phys. Rev. Lett. 62 (1989) 1833.

4. D. Guerreau, in Nuclear Matter and Heavy Ion Collisions, pp. 187, ed. M. Soyeur, H. Flocard, B. Tamain, and M. Porneuf, Plenum Publishing Corp (1989).

5. L. G. Moretto and G. J. Wozniak, Ann. Rev. Nucl \& Part. Sci., Vol. 43, (1993) in press. 

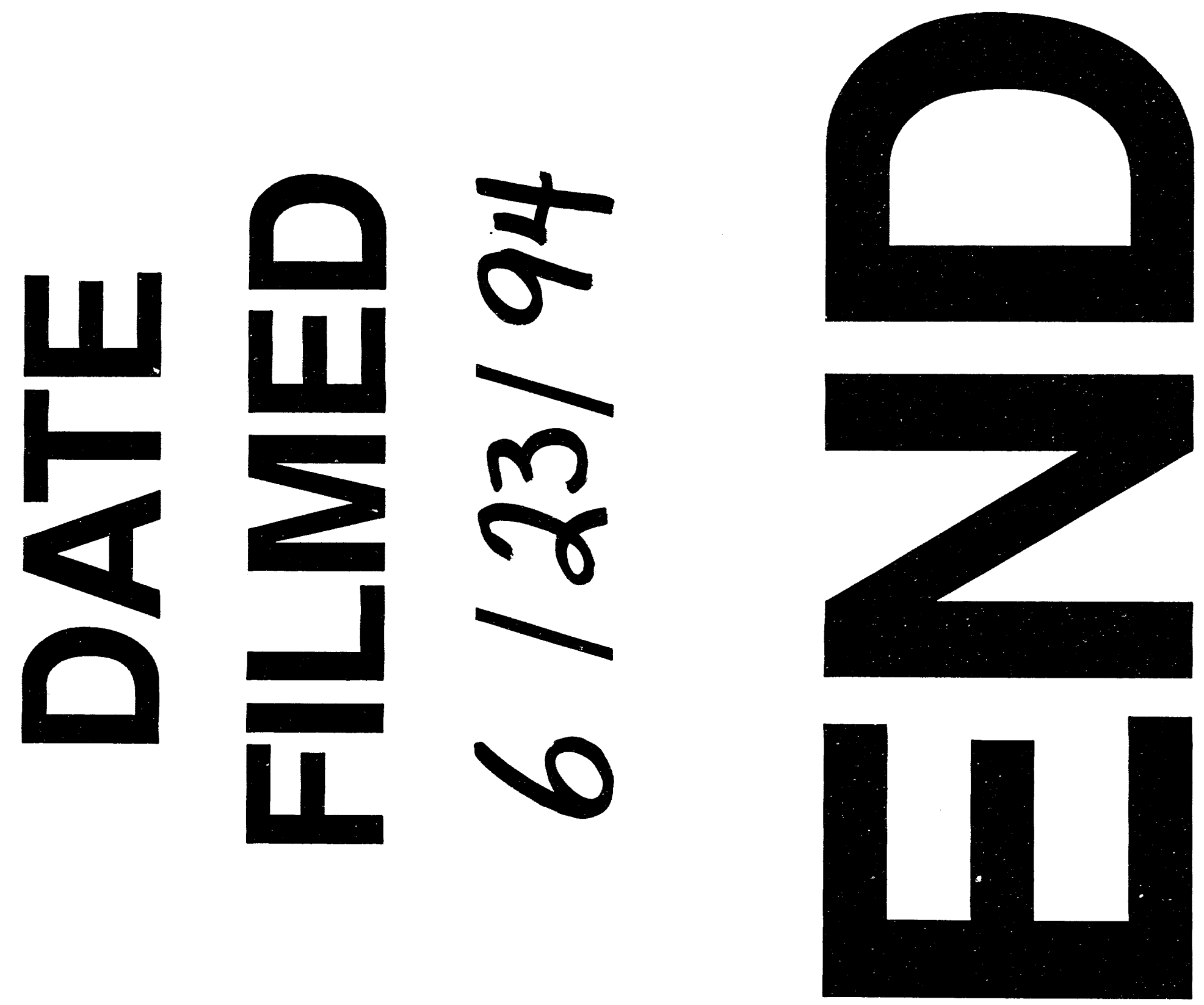


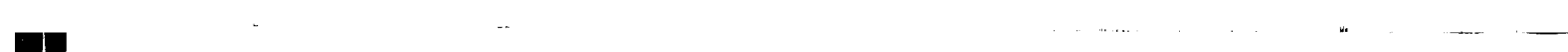

